Article

\title{
In Silico Analysis of the Antigenic Properties of Iron-Regulated Proteins against Neisseria meningitidis
}

\author{
Md. Shahedur Rahman ${ }^{1, *}{ }^{\dagger}$, Chayon Biswas ${ }^{1,+}$, Polash Kumar Biswas ${ }^{2,+}$, Md. Ashraful Kader ${ }^{1,3}$, \\ S. M. Nur Alam ${ }^{4}$, Christian Sonne ${ }^{5}$ (D) and Ki-Hyun Kim ${ }^{6, *}$ (D) \\ 1 Department of Genetic Engineering and Biotechnology, Jashore University of Science and Technology, \\ Jashore 7408, Bangladesh; chayonorgbd@gmail.com (C.B.); msb20001@stdmail.ump.edu.my (M.A.K.) \\ 2 Department of Stem Cell and Regenerative Biotechnology, Konkuk University, 120 Neungdong-ro, \\ Gwangjin-gu, Seoul 05029, Korea; polash93@konkuk.ac.kr \\ 3 Faculty of Industrial Sciences \& Technology, Universiti Malaysia Pahang, Kuantan 26300, Malaysia \\ 4 Department of Chemical Engineering, Jashore University of Science and Technology, \\ Jashore 7408, Bangladesh; smn.alam@just.edu.bd \\ 5 Department of Bioscience, Arctic Research Centre (ARC), Aarhus University, Frederiksborgvej 399, \\ P.O. Box 358, DK-4000 Roskilde, Denmark; cs@bios.au.dk \\ 6 Department of Civil and Environmental Engineering, Hanyang University, 222 Wangsimni-Ro, \\ Seoul 04763, Korea \\ * $\quad$ Correspondence: ms.rahman@just.edu.bd (M.S.R.); kkim61@hanyang.ac.kr (K.-H.K.) \\ + These authors contributed equally to this study.
}

Received: 8 August 2020; Accepted: 31 August 2020; Published: 3 September 2020

\begin{abstract}
Neisseria meningitidis is a commensal pathogen that causes infectious cerebrospinal disease in people of all ages. The multivariate role of six disease-causing polysaccharide serotypes is found to play a crucial role in developing vaccines (or general treatment strategies) to treat this emerging pathogen. Iron is a crucial transition metal for N. meningitidis. Proteomic analysis data could be valuable for vaccine design. Here, we conduct a comparative study using computational bioinformatic tools to identify the most effective iron-regulated outer membrane proteins (OMPs) as immunogenic targets for a potential vaccine against $N$. meningitidis. The basic properties of $N$. meningitidis OMPs are explored for flexibility, solubility, hydrophilicity, beta-turns, and overall antigenic probability. Results of our study suggest that iron-regulated OMPs are flexible and soluble in water with high densities of conformational B-cell epitopes. As such, they can be recommended as a novel candidate for a vaccine against $N$. meningitidis both in vitro and in vivo.
\end{abstract}

Keywords: Neisseria meningitidis; vaccine development; epidemic disease; immunogenicity

\section{Introduction}

Neisseria meningitidis is a diplococcal Gram-negative bacterial species that serves as the predominant causative infectious agent for a range of diseases classified as an invasive meningococcal disease [1]. Meningitis is defined as the infection of the meninges membranes encompassing the brain and spinal cord. N. meningitidis is an obligate human pathogen [2] and its infections may remain asymptomatic [3]. Meningitis progression is rapid and has complicated symptoms that make clinical diagnosis difficult and cause it to be a world-wide challenge to treat and prevent.

$N$. meningitidis is host-specific and adapted to sidestep the human immune system during pathogenesis as well as having commensal residence in the nasopharynx [2]. The human immune system is complex and can eliminate the meningococcus infection through an array of different strategies, including antimicrobial peptides and proteins, which play an important role in the innate immune system [4]. To combat the host antibacterial defense system, N. meningitidis modifies lipid 
A-groups of the toxins lipooligosaccharides through phosphoethanolamine [5]. The compounds present in the outer membrane increase meningococcal resistance against the human microbial peptide LL-37 [6]. As reported previously, a number of factors (e.g., biofilm formation ability, adherence to the cell, and the ability to invade host cells) are important for the virulence of this bacterium [7], while iron acquisition is another virulence factor and determinant of host cell death [8].

According to Centers for Disease Control and Prevention (CDC), there are two types of meningococcal vaccines available in the United States: meningococcal conjugate vaccine and serogroup $\mathrm{B}$ meningococcal vaccine. The former is recommended for 11 to 12 years old person while requiring to administer a booster dose after three to four years. On the other hand, the latter is for 10 years or older (https://www.cdc.gov/vaccines/vpd/mening/index.html). However, outer membrane protein vaccines have been observed to have high efficacy against group B in individuals over 4 years of age [9]. On the other hand, N. meningitidis is found to have low genomic stability. In light of all these factors, a stable, multi-purpose vaccine against $N$. meningitides is in urgent need for development.

Acquisition of iron is an important factor for the virulence of pathogens such as N. meningitidis [10]. If restricted, pathogenic bacteria require major transcriptional changes to amplify and transcript iron metabolic- and motility-related genes. Proteomic analysis may facilitate vaccine design through a better understanding of energy and metabolic processes as well as iron storage [11]. Proteomics bioinformatic tools are valuable for epitope prediction and vaccine design based on 3D proteomic structuring [12-14]. In the present study, we combine in silico analyses of the N. meningitidis genome structure with bioinformatic tools to identify potentially immunogenic antigens from $N$. meningitidis proteins. We verify the iron-regulated proteins from $N$. meningitidis and investigate the potential of iron-regulated proteins as candidates for the development of an N. meningitidis vaccine. Furthermore, our systematic selection and evaluation of antigens provide a new approach that will help lower the expense of cloning.

\section{Methods and Materials}

\subsection{Selection of Iron-Regulated Proteins of N. meningitidis}

For the in silico analysis of immunogenicity, outer membranes, iron-regulated proteins of N. meningitidis (CBA09441, CBA07476, CAM07737, and CBA03648), were selected through an NCBI search (http://www.ncbi.nlm.nih.gov/protein). All the protein sequences were retrieved from the NCBI server and saved in the FASTA format for subsequent analyses.

\subsection{Antigenicity and Solubility Prediction}

We used the Immune Epitope Database (IEDB, http://tools.immuneepitope.org) to predict the average scores for flexibility, hydrophilicity, and beta turns of proteins [15]. We used VaxiJen (alignment-independent prediction of protective antigens) (http://www.ddg-pharmfac.net/vaxijen/ VaxiJen/VaxiJen.html) [16] and ANTIGENpro (http://scratch.proteomics.ics.uci.edu/) to analyze the antigenic probability. The threshold parameter for VaxiJen was estimated at $0.4(40 \%)$. In ANTIGENpro, the protein antigenicity was predicted based on five machine learning approaches followed by a Support Vector Machine (SVM) classifier. The solubilities of the selected antigenic proteins were predicted with SOLpro (http://scratch.proteomics.ics.uci.edu) using a two-stage support vector machine architecture [17].

\subsection{Prediction of Linear and Conformational B-Cell Epitopes}

The ABCpred webserver (http://www.imtech.res.in/raghava/abcpred) was used to provide a linear B-cell epitope prediction using a recurrent neural network algorithm trained with BciPep and SwissProt databases (https://www.ebi.ac.uk/) that contained 700 experimentally detected B-cell epitopes. The best results were acquired with the aid of a window length of 16 residues [18] with a threshold for predictions of 0.80 (i.e., without an epitope overlapping filter). BCpreds (http://ailab.cs.iastate. edu/bcpreds/predict.html) was employed to predict overlapping epitopes with $80 \%$ specificity for 
flexible length, linear B-cell epitopes [19]. BepiPred (http://www.cbs.dtu.dk/services/BepiPred) was utilized to additionally predict B-cell epitopes from antigen sequences (threshold parameter as 0.8 $(80 \%)$ ), while ElliPro (http://tools.immuneepitope.org/tools/ElliPro/iedb) predicted both linear and conformational B-cell epitopes. Compared to six other structure-based methods that can be used for epitope prediction, ElliPro was reported to perform the best [20].

We also explored DiscoTope (http://www.cbs.dtu.dk/services/DiscoTope/) as a novel tool to predict conformational B-cell epitopes from three-dimensional structural data of the protein with the aid of spatial information, amino acid statistics, and surface accessibility. The predictions were used for the compilation of discontinuous epitopes as ascertained by X-ray crystallography of antibody/antigen protein complexes [21]. In addition, we predicted conformational epitopes based on the Ellipro server. To predict conformational B cell epitope in ElliPro, its prediction parameter was selected in the range of 0.5 to 8 . Peptides that were predicted to be epitopes were selected by their high epitope density.

\subsection{Template Identification and Comparative Model Building}

Comparative protein modeling is an alternative for experimental models based on the SWISS-MODEL template library. The best performing model was selected for further analysis and protein comparative modeling of the target sequences was done using the SWISS-MODEL server (https://swissmodel.expasy.org/), after which the PyMOL software was employed to visualize the results of the 3D protein models [22-24]. Ray tracing was also performed to generate a better view of the 3D models of these proteins.

\subsection{Evaluation of Comparative Model}

The protein models generated through the SWISS-MODEL server were extracted into the PDBsum server for the purpose of Ramachandran plot analysis. The in silico stereochemical quality and accuracy were analyzed by PROCHECK [25]. After that, the protein model selected based on its discrete optimized protein energy (DOPE) score was analyzed by GROMOS96 of Swiss-PdbViewer 4.1.0 software to minimize the energy [26]. Note that Steepest Descent algorithm was employed to assess the lowest energy conformation for the predicted protein molecules. Parameters for steps and cut off were selected as 20 and 10 angstrom, respectively. Further, the energy-minimized models were put into the SAVES server as a means to verify the 3D results (http://nihserver.mbi.ucla.edu/SAVES) for ERRAT. ERRAT was used to verify the protein structures on the basis of an empirical, atom-based approach [27]. In this work, the ProSA server was utilized to evaluate the protein models. Finally, Verify_3D was utilized to assess the protein model and three-dimensional profiling [28].

\section{Results}

\subsection{Antigenicity and Solubility Prediction}

We started our investigation with five proteins (Supplementary Table S1). Among them, as four proteins (CBA09441, CBA07476, CAM07737, and CBA03648) showed high antigenicity scores (Table 1), they were selected for further analysis. According to the IEDB server, the hydrophilicity scores ranged from 2.167 to 2.18. In the case of flexibility, CBA09441, CAM07737, and CBA03648 scored 1.007 and CBA07476 scored 1.006. For the beta turns, CBA09441, CBA07476, and CAM07737 scored 1.025 and CBA03648 scored 1.029. In contrast, the antigenicity prediction scores from the VaxiJen server ranged from 0.6939 to 0.7186 and the ANTIGENpro server scores ranged from 0.90866 to 0.9154 (Table 1). These results indicate that those proteins possess the structural flexibility and immunogenic properties. In this study, CBA07476, CAM07737, CBA03648, and CBA09441 were soluble in SOLpro analysis, whereas CBA09441 was soluble in SOLpro with the highest SOLpro probability of 0.823523 (Table 2). 
Table 1. Antigenic and immunogenic properties of proteins.

\begin{tabular}{|c|c|c|c|c|c|}
\hline Accession No. & & IEDB & & VaxiJen & ANTIGENPro \\
\hline & Hydrophilicity & Flexibility & Beta Turn & & \\
\hline CBA09441 & 2.176 & 1.007 & 1.025 & 0.7022 & 0.909208 \\
\hline CBA07476 & 2.167 & 1.006 & 1.025 & 0.7186 & 0.90866 \\
\hline CAM07737 & 2.18 & 1.007 & 1.025 & 0.7092 & 0.910421 \\
\hline CBA03648 & 2.177 & 1.007 & 1.029 & 0.6939 & 0.9154 \\
\hline
\end{tabular}

Table 2. Solubility of proteins.

\begin{tabular}{cc}
\hline Accession No. & \multicolumn{1}{c}{ SOLpro } \\
\hline CBA09441 & SOLUBLE with probability 0.823523 \\
CBA07476 & SOLUBLE with probability 0.756444 \\
CAM07737 & SOLUBLE with probability 0.713970 \\
CBA03648 & SOLUBLE with probability 0.805877 \\
\hline
\end{tabular}

\subsection{Linear and Conformational B-Cell Epitopes Prediction}

According to ABCpred, the linear B-cell epitope densities for all the proteins ranged from 0.49 to 0.55 . CAM07737 predicted by the BepiPred server showed high linear B-cell epitope densities. In the case of the BCpred server, CAM07737 and CBA07476 showed high epitope densities. Additionally, the ElliPro server also predicted CBA07476 with high epitope densities relative to other proteins (Table 3).

Table 3. Linear B-cell epitope densities of proteins.

\begin{tabular}{cccccccccc}
\hline \multirow{2}{*}{ Accession No. } & Length & \multicolumn{2}{c}{ ABCpred } & \multicolumn{2}{c}{ BepiPred } & \multicolumn{2}{c}{ BCpred } & \multicolumn{2}{c}{ Ellipro } \\
\cline { 2 - 9 } & & EN & ED & EN & ED & EN & ED & EN & ED \\
\hline CBA09441 & 713 & 352 & 0.49 & 149 & 0.20 & 140 & 0.19 & 216 & 0.30 \\
CBA07476 & 721 & 368 & 0.51 & 143 & 0.19 & 160 & 0.22 & 226 & 0.31 \\
CAM07737 & 714 & 368 & 0.52 & 157 & 0.21 & 160 & 0.22 & 206 & 0.28 \\
CBA03648 & 723 & 400 & 0.55 & 148 & 0.20 & 140 & 0.19 & 219 & 0.30 \\
\hline
\end{tabular}

$\mathrm{EN}=$ Epitope starting residue number, $\mathrm{ED}=$ Epitope density.

The DiscoTope server analysis resulted in a list of proteins (e.g., CBA09441, CBA07476, CAM07737, and CBA03648) with conformational epitope density scores of $0.49,0.49,0.43$, and 0.50 , respectively, which was different from the Ellipro server scores of $0.48,0.47,0.50$, and 0.48 , respectively. It was demonstrated based on the DiscoTope server that CBA03648 should have a high conformational epitope density. With the Ellipro server, CAM07737 also exhibited high densities of conformational B-cell epitopes (e.g., relative to other proteins), as shown in Table 4.

Table 4. Conformational B-cell epitope densities of proteins predicted with DiscoTope, and ElliPro.

\begin{tabular}{cccccc}
\hline \multirow{2}{*}{ Accession No. } & \multicolumn{2}{c}{ DiscoTope } & \multicolumn{2}{c}{ Ellipro } & \multirow{2}{*}{ Length } \\
\cline { 2 - 5 } & EN & ED & EN & ED & \\
\hline CBA09441 & 351 & 0.49 & 343 & 0.48 & 713 \\
CBA07476 & 354 & 0.49 & 342 & 0.47 & 721 \\
CAM07737 & 313 & 0.43 & 357 & 0.50 & 714 \\
CBA03648 & 360 & 0.50 & 349 & 0.48 & 723 \\
\hline
\end{tabular}

$\mathrm{EN}=$ Epitope residue number, $\mathrm{ED}=$ Epitope density. 


\subsection{Templates Analyses, Comparative Protein Model Building, and Visualization}

Figure 1 presents the predicted protein models. CAM07737, CBA03648, and CBA09441 possess $\mathrm{Fe}^{+3}$ molecules as ligand in their structure. The initial energy values of the proteins were as follows: CBA09441 $(-33,787.887 \mathrm{Kcal} / \mathrm{mol})$, CBA07476 $(-34,049.195 \mathrm{Kcal} / \mathrm{mol})$, CAM07737 $(-30,584.434 \mathrm{Kcal} / \mathrm{mol})$, and CBA03648 $(-31,806.014 \mathrm{Kcal} / \mathrm{mol})$. After energy minimization the energy values were $-43,611.754 \mathrm{Kcal} / \mathrm{mol},-43,311.914 \mathrm{Kcal} / \mathrm{mol},-41,019.699 \mathrm{Kcal} / \mathrm{mol}$, and $-41,996.238 \mathrm{Kcal} / \mathrm{mol}$, respectively (Table 5).

A

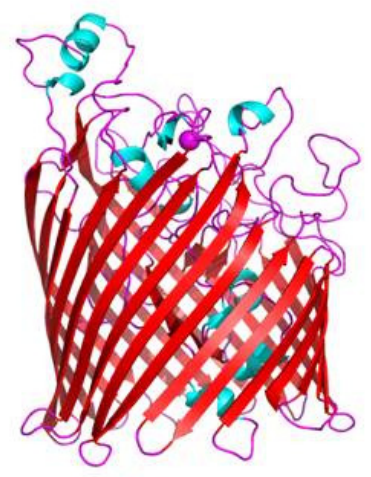

C

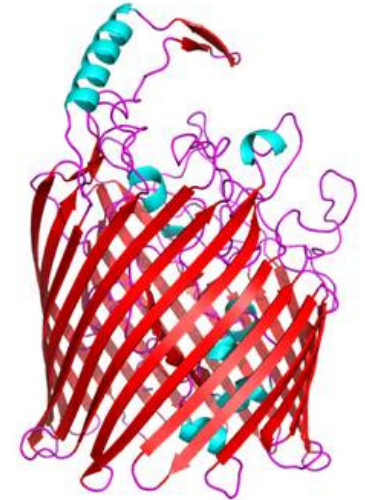

B

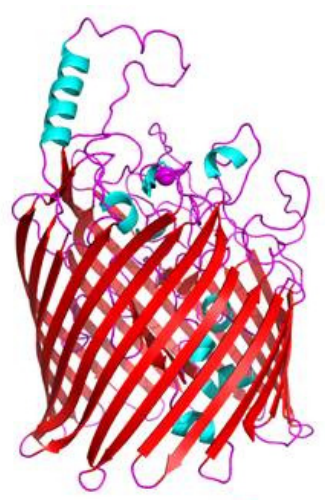

D

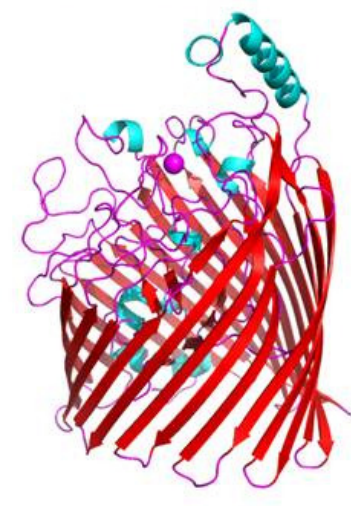

Figure 1. Predicted 3D protein models. (A) CAM07737. (B) CBA03648. (C) CBA07476. (D) CBA09441 (FrpB). The ball structure (magenta color) represents the $\mathrm{Fe}^{+3}$ ligand molecule.

Table 5. Model evaluation scores and energy of the model of before and after energy minimization.

\begin{tabular}{cccccc}
\hline Accession No. & $\begin{array}{c}\text { ProSA } \\
\text { (Z-Score) }\end{array}$ & $\begin{array}{c}\text { Verify_3D } \\
\text { Score (\%) }\end{array}$ & $\begin{array}{c}\text { ERRAT } \\
\text { Value }\end{array}$ & $\begin{array}{c}\text { Energy of the Model } \\
\text { before Energy } \\
\text { Minimization } \\
\text { (Kcal/mol) }\end{array}$ & $\begin{array}{c}\text { Energy of the } \\
\text { Model after Energy } \\
\text { Minimization } \\
\text { (Kcal/mol) }\end{array}$ \\
\hline CBA09441 & -4.92 & 80.42 & 91.6667 & $-33,787.887$ & $-43,611.754$ \\
CBA07476 & -4.89 & 81.7 & 86.5067 & $-34,049.195$ & $-43,311.914$ \\
CAM07737 & -4.55 & 80.3 & 91.0334 & $-30,584.434$ & $-41,019.699$ \\
CBA03648 & -5.19 & 80.41 & 92.0777 & $-31,806.014$ & $-41,996.238$ \\
\hline
\end{tabular}

\subsection{Evaluation of Predicted Protein Models}

CBA09441, CAM07737, and CBA03648 had good G-score values (Table 6, Figure 2). The G-factor is used as a measure of a given stereochemical property in terms of normality. For a low G-factor, the property should have a low-probability conformation. The analysis of protein models revealed that CBA09441, CBA07476, CAM07737, and CBA03648 had good Verify_3D scores of 80.42, 81.7, 80.3, and $80.41 \%$, respectively (Table 5 ). 
Table 6. Ramachandran plot statistics and overall G-factors of N. meningitidis proteins.

\begin{tabular}{ccccc}
\hline & \multicolumn{3}{c}{ Ramachandran Plot Statistics } & \\
\cline { 2 - 4 } Accession No. & $\begin{array}{c}\text { Most Favored } \\
\text { Regions }\end{array}$ & $\begin{array}{c}\text { Additional } \\
\text { Allowed Regions }\end{array}$ & $\begin{array}{c}\text { Generously } \\
\text { Allowed Regions }\end{array}$ & Overall G-Factor \\
\hline CBA09441 & 502 & 63 & 1 & -0.35 \\
CBA07476 & 531 & 55 & 4 & -0.21 \\
CAM07737 & 502 & 63 & 1 & -0.35 \\
CBA03648 & 502 & 63 & 1 & -0.35 \\
\hline
\end{tabular}
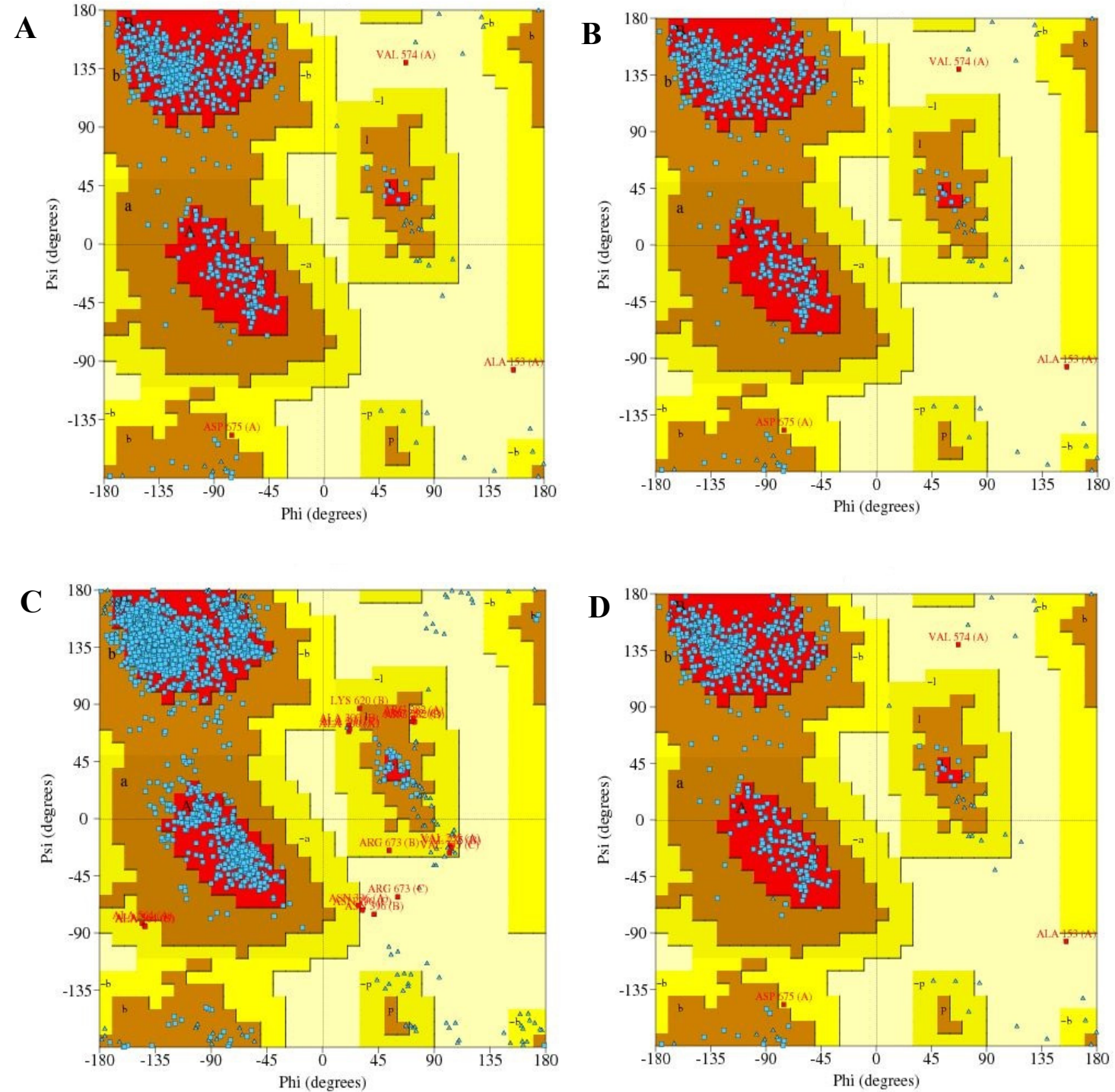

Figure 2. Ramachandran plot of predicted protein models. (A) CAM07737 (714 amino acids). (B) CBA03648 (723 amino acids). (C) CBA07476 (721 amino acids). (D) CBA09441 (713 amino acids). The residues in red zone indicate their position in most favored regions.

According to the ERRAT value, all protein models showed scores ranging from 86.5 to 92 (Table 5). ERRAT value can be used to statistically assess non-bonded interactions between different atom types. The accuracy of the predicted protein model was confirmed by a negative Z-score value (Figure 3). The Z-score can be used to judge the overall model quality based on the evaluation of the deviation of the total energy in the structure in relation to an energy distribution derived from random 
conformations [29]. The Z-scores of the four experimentally predicted protein models (i.e., CBA09441, CBA07476, CAM07737, and CBA03648) were $-4.92,-4.89,-4.55$, and -5.19 , respectively.

A
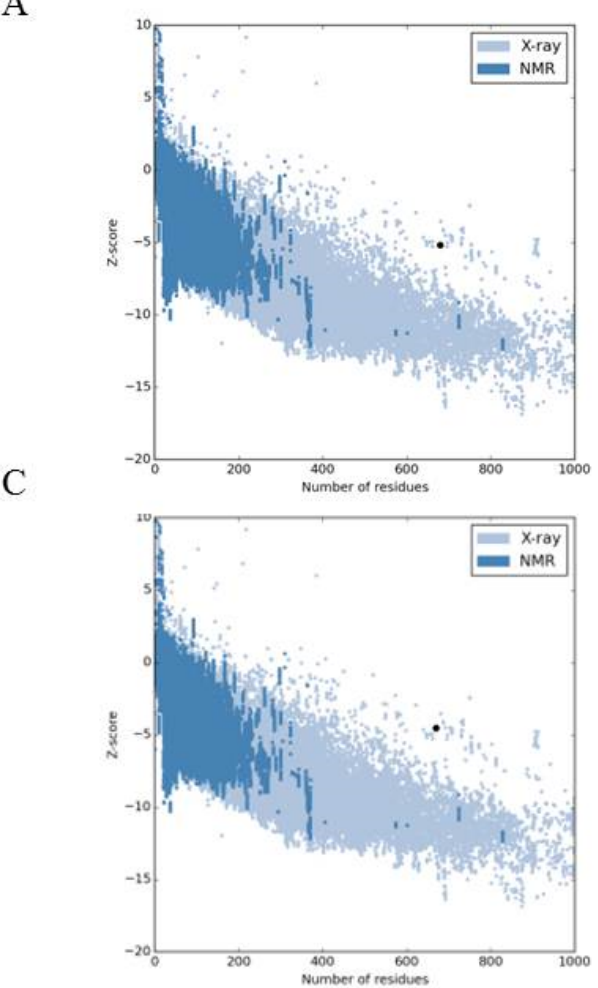

B
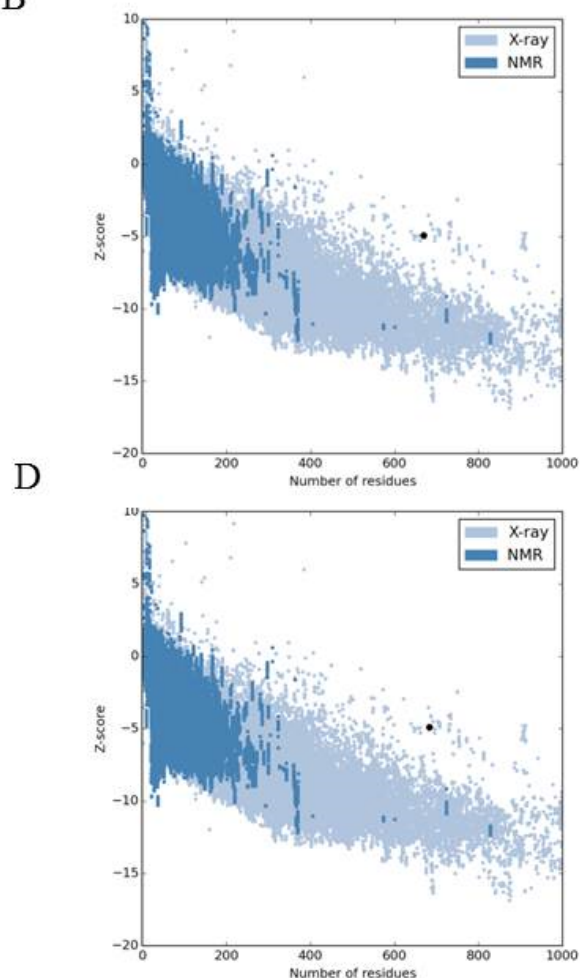

Figure 3. Overall model quality of predicted protein models by ProSA. (A) CAM07737. (B) CBA03648.

(C) CBA07476. (D). CBA09441.

\section{Discussion}

As a human pathogen, N. meningitidis is known to cause devastating effect on humans [30]. The expression of several genes in response to iron (e.g., specific virulence factors) was reported to be regulated by the pathogenic Neisseria. As all bacteria demands iron for their growth, immunogenicity analysis for vaccine and drug design often sets iron-regulated $N$. meningitidis protein as the target [31]. To develop new vaccines (or new therapies) for undetectable bacterial agents in the pathogenesis, it is often crucial to find new cell-surface and/or secreted proteins [32]. Outer membrane proteins may have a strong immunogenic property with easy accessibility to antibodies [33,34]. In the host, the infection and survival of the N. meningitidis is siderophore-dependent [35]. Neisseria species hijack siderophore produced by other bacteria [36]. FrpB is an integral outer membrane protein from N. meningitides. It was demonstrated that this protein should possess antigeic properties as a potential candidate for a vaccine against meningococcal meningitis [37-39]. This protein has been extensively characterized previously $[37,40]$. FrpB promotes the uptake of iron across the outer membrane to work as siderophore receptor $[37,38]$. Thus, because of their outer membrane localization, iron-regulated proteins are easily exposed to the host immune system, which responds to the immunogenicity and protection probability by provoking higher antibody production [41,42]. In other bacterial species, the use of specific antibodies against iron-regulated outer membrane proteins (IROMPs) is for bactericidal purposes to inhibit the uptake of iron. [43].

Nuclear magnetic resonance (NMR) and X-ray crystallography methods have been utilized to determine the 3D structure of proteins [44,45]. However, these methods are time-consuming and highly expensive. We used comparative homology modeling instead of experimental NMR and X-ray crystallography. Comparative homology modeling is considered as a highly favorable option 
because of its capability for function prediction as well as its application in different research fields (e.g., 3D structure prediction, mutagenesis analysis, and disulfide bridge analysis) [46,47]. We predicted 3D structures for all four IROMPs and validated them. The continuous decrease of force field energy was realized through energy minimization after the refinement of the protein model. Our data also suggested that, in terms of the Z-score, the $G$ factor value and ERRAT value of all four IROMPs were good, but among them, CBA03648 had the highest score.

In the current study, IROMPs from N. meningitidis were chosen to estimate their immunogenicity for the generation of a vaccine. The immunogenicity of an antigen is reported to be affected by several properties, such as mobility of backbone atoms, accessibility, flexibility, hydrophilicity, and topology [48]. All data obtained from Vaxijen, ANTIGENpro, and IEDB were comparable to each other considering the lowest scores for antigenicity. Protein solubility is another important factor used for immunogenicity prediction. Insoluble proteins are not appropriate for an immunogenicity effect. We used SOLpro to predict the solubility of protein. SOLpro provides the corresponding solubility probability (above 0.5 ) (http://scratch.proteomics.ics.uci.edu/explanation.html\#SOLpro). Our data suggest that all of the predicted protein models should be water-soluble (Table 2).

An essential task in the design of a vaccine is to choose proteins with antibody-binding epitopes (B-cell epitopes) through which the immune response can be induced efficiently [49,50]. All of our predicted protein models showed a high B-cell epitope density that could be grouped into two classes: linear or conformational epitopes. The former are short peptides while the latter are composed of amino acids that are within the structure of a folded 3D protein [51]. In our study, we predicted linear and conformational B-cell epitopes with the aid of several bioinformatics tools that contributed to the elevated accuracy of the prediction. All these bioinformatics tools were highly useful in predicting that CBA03648 should possess large amounts of linear and conformational B-cell epitopes relative to other proteins. It is noted that antigenicity and immunogenicity of the protein can increase significantly with increases in epitope density in a single protein molecule [52]. As such, it can be concluded that CBA03648 is a better vaccine candidate than the other three proteins.

In this research, we conducted a comparative assessment on the immunogenic and antigenic differences between protein molecules that exhibit either high or low degrees of epitope density. Accordingly, all the predicted protein models were seen to have a high epitope density. However, among the four IROMPs, as mentioned earlier, CBA03648 had better epitope density (ABCpred score 0.55 and Ellipro score 0.30). High epitope density in a protein molecule leads to a significant enhancement of immunogenicity and antigenicity [52,53]. Both protective immunity and epitope density are useful for revealing vital pieces of information for vaccine development. Further, the recombinant proteins of higher epitope densities were identified to contribute greatly to the enhancement of survival rates for mice [54]. The results of the in silico study suggested that all four proteins should contribute significantly to siderophore-mediated iron uptake. Hence, they can exert a greater immunogenicity effect on humans. A similar kind of in silico study is conducted by Bazmara et al., in 2017. They did the investigation on iron-regulated proteins in Acinetobacter baumannii [55]. They find that CarO is an efficient immunogenic protein in A. baumannii. A similar in silico study was conducted by Adhikari and Rahman 2017, with similar outcomes [56].

The protein model selected in our work (CBA09441, CAM07737, and CBA03648) recorded an ERRAT score of above $91 \%$ to indicate a low resolution of our model [57]. Although our model has low resolutions, similar results were also reported previously [57-59]. The protein model in this work showcased a good negative Z-score value similar to others [56]. On the other hand, Verify_3D score of a predicted model should be more than $80 \%$ [60]. All of our predicted models showed Verify_3D score above $80 \%$ to prove good quality. In the case of the Ramachandran plot, above $90 \%$ of amino acid residues should be in the most favored region for a modeled structure [57]. Our data are also found to fulfill this criterion when tested using those predicted protein models. Thus, the overall scores of all the predicted protein models are quite reliable and satisfactory. 


\section{Conclusions}

Together with bioinformatic tools, we validated our protein models with respect to stereochemical quality, protein model accuracy, its resolution, and our predicted model quality. Our in silico modeling showed that iron-regulated proteins from $N$. meningitidis had the potential to cause an immune response in humans. These proteins are expected to maintain high densities of conformational B-cell epitopes, high solubility, and high antigenicity. Thus, our results help to better conduct in vitro and in vivo research in the field of vaccine design.

Supplementary Materials: The following are available online at http://www.mdpi.com/2076-3417/10/17/6113/s1, Table S1: Antigenic and immunogenic properties of proteins.

Author Contributions: M.S.R. and C.B. performed data analysis and drafted the manuscript. P.K.B. organized data and wrote and edited the manuscript. M.A.K. and S.M.N.A. collected and analyzed data. C.S. takes part in drafting manuscript. K.-H.K. analyzed data and wrote the manuscript. M.S.R. reorganized the data, edited the manuscript design, and supervised the study. All authors have read and agreed to the published version of the manuscript.

Funding: This research is partially funded by National Research Foundation of Korea (NRF) funded by the Ministry of Science, ICT \& Future Planning (Grant No: 2016R1E1A1A01940995).

Acknowledgments: The corresponding author (K.-H.K.) acknowledges support made in part by grants from the National Research Foundation of Korea (NRF) funded by the Ministry of Science, ICT \& Future Planning (Grant No: 2016R1E1A1A01940995). M.S.R. acknowledges support of the Ministry of Science and Technology (R \& D project), Bangladesh.

Conflicts of Interest: The authors declare no conflict of interest.

\section{References}

1. Stephens, D.S.; Greenwood, B.; Brandtzaeg, P. Epidemic meningitis, meningococcaemia, and Neisseria meningitidis. Lancet 2007, 369, 2196-2210. [CrossRef]

2. Yazdankhah, S.P.; Caugant, D.A. Neisseria meningitidis: An overview of the carriage state. J. Med. Microbiol. 2004, 53, 8218-8232. [CrossRef]

3. Walayat, S.; Hussain, N.; Malik, A.H.; Vazquez-Melendez, E.; Aulakh, B.S.; Lynch, T. Invasive meningococcal disease without meningitis: A forgotten diagnosis. Int. Med. Case Rep. J. 2018, 11, 87-90. [CrossRef]

4. Lo, H.; Tang, C.M.; Exley, R.M. Mechanisms of avoidance of host immunity by Neisseria meningitidis and its effect on vaccine development. Lancet Infect. Dis. 2009, 9, 418-427. [CrossRef]

5. Tzeng, Y.-L.; Stephens, D.S. Antimicrobial peptide resistance in Neisseria meningitidis. Biochim. Biophys. Acta (BBA) Biomembr. 2015, 1848, 3026-3031. [CrossRef]

6. Tzeng, Y.-L.; Ambrose, K.D.; Zughaier, S.; Zhou, X.; Miller, Y.K.; Shafer, W.M.; Stephens, D.S. Cationic antimicrobial peptide resistance in Neisseria meningitidis. J. Bacteriol. 2005, 187, 5387-5396. [CrossRef]

7. Yi, K.; Rasmussen, A.W.; Gudlavalleti, S.K.; Stephens, D.S.; Stojiljkovic, I. Biofilm formation by Neisseria meningitidis. Infect. Immun. 2004, 72, 6132-6138. [CrossRef] [PubMed]

8. Jin, J.S.; Kwon, S.-O.; Moon, D.C.; Gurung, M.; Lee, J.H.; Kim, S.I.; Lee, J.C. Acinetobacter baumannii Secretes Cytotoxic Outer Membrane Protein A via Outer Membrane Vesicles. PLoS ONE 2011, 6, e17027. [CrossRef]

9. Frasch, C.E.; Bash, M.C.; Ellis, R.W.; Brodeur, B.R. Neisseria meningitidis Vaccines. In New Bacterial Vaccines; Springer Science and Business Media LLC: Berlin/Heidelberg, Germany, 2003; pp. 229-243.

10. Tong, Y.; Guo, M. Bacterial heme-transport proteins and their heme-coordination modes. Arch. Biochem. Biophys. 2009, 481, 1-15. [CrossRef]

11. Rinaudo, C.D.; Telford, J.L.; Rappuoli, R.; Seib, K.L. Vaccinology in the genome era. J. Clin. Investig. 2009, 119, 2515-2525. [CrossRef]

12. Jahangiri, A.; Rasooli, I.; Gargari, S.L.M.; Owlia, P.; Rahbar, M.R.; Amani, J.; Khalili, S. An in silico DNA vaccine against Listeria monocytogenes. Vaccine 2011, 29, 6948-6958. [CrossRef] [PubMed]

13. Khalili, S.; Rahbar, M.R.; Dezfulian, M.H.; Jahangiri, A. In silico analyses of Wilms' tumor protein to designing a novel multi-epitope DNA vaccine against cancer. J. Theor. Biol. 2015, 379, 66-78. [CrossRef] [PubMed]

14. Mohammadpour, H.; Pourfathollah, A.A.; Zarif, M.N.; Khalili, S. Key role of Dkk3 protein in inhibition of cancer cell proliferation: An in silico identification. J. Theor. Boil. 2016, 393, 98-104. [CrossRef] 
15. Zhang, Q.; Wang, P.; Kim, Y.; Haste-Andersen, P.; Beaver, J.; Bourne, P.E.; Bui, H.-H.; Buus, S.; Frankild, S.; Greenbaum, J.; et al. Immune epitope database analysis resource (IEDB-AR). Nucleic Acids Res. 2008, 36, W513-W518. [CrossRef]

16. Doytchinova, I.A.; Flower, D.R. VaxiJen: A server for prediction of protective antigens, tumour antigens and subunit vaccines. BMC Bioinform. 2007, 8, 4. [CrossRef]

17. Magnan, C.N.; Randall, A.; Baldi, P. SOLpro: Accurate sequence-based prediction of protein solubility. Bioinformatics 2009, 25, 2200-2207. [CrossRef]

18. Reimer, U. Prediction of linear B-cell epitopes. In Epitope Mapping Protocols; Springer: Berlin/Heidelberg, Germany, 2009; pp. 335-344.

19. El-Manzalawy, Y.; Dobbs, D.; Honavar, V.G. Predicting linear B-cell epitopes using string kernels. J. Mol. Recognit. 2008, 21, 243-255. [CrossRef]

20. Ponomarenko, J.; Bui, H.-H.; Li, W.; Fusseder, N.; Bourne, P.E.; Sette, A.; Peters, B. ElliPro: A new structure-based tool for the prediction of antibody epitopes. BMC Bioinform. 2008, 9, 514. [CrossRef]

21. Kringelum, J.V.; Lundegaard, C.; Lund, O.; Nielsen, M. Reliable B Cell Epitope Predictions: Impacts of Method Development and Improved Benchmarking. PLoS Comput. Boil. 2012, 8, e1002829. [CrossRef]

22. DeLano, W.L. PyMol: An open-source molecular graphics tool. CCP4 Newsl. Protein Crystallogr. 2002, 40, $82-92$.

23. Samad, A.; Ahammad, F.; Nain, Z.; Alam, R.; Imon, R.R.; Hasan, M.; Rahman, S. Designing a multi-epitope vaccine against SARS-CoV-2: An immunoinformatics approach. J. Biomol. Struct. Dyn. 2020, 2020, 1-17. [CrossRef]

24. Barman, U.D.; Saha, S.K.; Kader, A.; Jamal, M.A.H.M.; Sharma, S.P.; Samad, A.; Rahman, S. Clinicopathological and prognostic significance of GPC3 in human breast cancer and its 3D structure prediction. Netw. Model. Anal. Heal. Inf. Bioinform. 2020, 9, 1-18. [CrossRef]

25. Laskowski, R.A.; Rullmann, J.A.C.; MacArthur, M.W.; Kaptein, R.; Thornton, J.M. AQUA and PROCHECK-NMR: Programs for checking the quality of protein structures solved by NMR. J. Biomol. NMR 1996, 8, 477-486. [CrossRef]

26. Guex, N.; Peitsch, M.C. SWISS-MODEL and the Swiss-Pdb Viewer: An environment for comparative protein modeling. Electrophoresis 1997, 18, 2714-2723. [CrossRef]

27. Colovos, C.; Yeates, T.O. Verification of protein structures: Patterns of nonbonded atomic interactions. Protein Sci. 1993, 2, 1511-1519. [CrossRef]

28. Lüthy, R.; Bowie, J.U.; Eisenberg, D. Assessment of protein models with three-dimensional profiles. Nature 1992, 356, 83-85. [CrossRef]

29. Wiederstein, M.; Sippl, M.J. ProSA-web: Interactive web service for the recognition of errors in three-dimensional structures of proteins. Nucleic Acids Res. 2007, 35, W407-W410. [CrossRef]

30. Pizza, M.; Rappuoli, R. Neisseria menin gitidis: Pathogenesis and immunity. Curr. Opin. Microbiol. 2015, 23, 68-72. [CrossRef]

31. Mathieu, B.; Pierre, C.; Christine, B. Iron Metabolism: A Promising Target for Antibacterial Strategies. Recent Pat. Anti Infect. Drug Discov. 2009, 4, 190-205.

32. Grifantini, R.; Sebastian, S.; Frigimelica, E.; Draghi, M.; Bartolini, E.; Muzzi, A.; Rappuoli, R.; Grandi, G.; Genco, C.A. Identification of iron-activated and -repressed Fur-dependent genes by transcriptome analysis of Neisseria meningitidis group B. Proc. Natl. Acad. Sci. USA 2003, 100, 9542-9547. [CrossRef]

33. Gao, Z.; Ye, C.; Zhou, L.; Zhang, Y.; Ge, Y.; Chen, W.; Pan, J. Evaluation of the $\beta$-barrel outer membrane protein VP1243 as a candidate antigen for a cross-protective vaccine against Vibrio infections. Microb. Pathog. 2020, 147, 104419. [CrossRef]

34. Bunikis, J.; Barbour, A.G. Access of Antibody or Trypsin to an Integral Outer Membrane Protein (P66) of Borrelia burgdorferi Is Hindered by Osp Lipoproteins. Infect. Immun. 1999, 67, 2874-2883. [CrossRef] [PubMed]

35. Pintor, M.; Ferrón, L.; Gomez, J.A.; Gorringe, A.; Criado, M.T.; Ferreirós, C.M. Blocking of iron uptake by monoclonal antibodies specific for the Neisseria meningitidis transferrin-binding protein 2. J. Med. Microbiol. 1996, 45, 252-257. [CrossRef] [PubMed]

36. Cornelissen, C.N. Subversion of nutritional immunity by the pathogenic Neisseriae. Pathog. Dis. 2017, 76, 112. [CrossRef] 
37. Saleem, M.; Prince, S.M.; Patel, H.; Chan, H.; Feavers, I.M.; Derrick, J.P. Refolding, purification and crystallization of the FrpB outer membrane iron transporter from Neisseria meningitidis. Acta Crystallogr. Sect. F Struct. Boil. Cryst. Commun. 2012, 68, 231-235. [CrossRef]

38. Kortekaas, J.; Müller, S.A.; Ringler, P.; Gregorini, M.; Weynants, V.E.; Rutten, L.; Bos, M.P.; Tommassen, J. Immunogenicity and structural characterisation of an in vitro folded meningococcal siderophore receptor (FrpB, FetA). Microbes Infect. 2006, 8, 2145-2153. [CrossRef] [PubMed]

39. Alaaldeen, D.; Davies, H.; Borriello, S. Vaccine potential of meningococcal FrpB: Studies on surface exposure and functional attributes of common epitopes. Vaccine 1994, 12, 535-541. [CrossRef]

40. Beucher, M.; Sparling, P.F. Cloning, sequencing, and characterization of the gene encoding FrpB, a major iron-regulated, outer membrane protein of Neisseria gonorrhoeae. J. Bacteriol. 1995, 177, 2041-2049. [CrossRef]

41. Sood, S.; Rishi, P.; Dhawan, V.; Sharma, S.; Ganguly, N.K. Protection mediated by antibodies to iron-regulated outer-membrane proteins of S. typhi in a mouse peritonitis model. Mol. Cell. Biochem. 2005, 273, 69-78. [CrossRef]

42. Misra, N.; Pu, X.; Holt, D.N.; McGuire, M.A.; Tinker, J.K. Immunoproteomics to identify Staphylococcus aureus antigens expressed in bovine milk during mastitis. J. Dairy Sci. 2018, 101, 6296-6309. [CrossRef]

43. Goel, V.K.; Kapil, A. Monoclonal antibodies against the iron regulated outer membrane proteins of Acinetobacter baumannii are bactericidal. BMC Microbiol. 2001, 1, 16. [CrossRef]

44. Hoffman, D.W.; Cameron, C.S.; Davies, C.; White, S.W.; Ramakrishnan, V. Ribosomal Protein L9: A Structure Determination by the Combined Use of X-ray Crystallography and NMR Spectroscopy. J. Mol. Boil. 1996, 264, 1058-1071. [CrossRef] [PubMed]

45. Grisshammer, R. New approaches towards the understanding of integral membrane proteins: A structural perspective on G protein-coupled receptors. Protein Sci. 2017, 26, 1493-1504. [CrossRef] [PubMed]

46. Gromiha, M.M.; Nagarajan, R.; Selvaraj, S. Protein Structural Bioinformatics: An Overview. In Encyclopedia of Bioinformatics and Computational Biology; Elsevier BV: Amsterdam, The Netherlands, 2019; pp. 445-459.

47. Skariyachan, S.; Garka, S. Chapter 1-Exploring the binding potential of carbon nanotubes and fullerene towards major drug targets of multidrug resistant bacterial pathogens and their utility as novel therapeutic agents. In Fullerens, Graphenes and Nanotubes; Grumezescu, A.M., Ed.; William Andrew Publishing: Norwich, NY, USA, 2018; pp. 1-29.

48. Kim, M.; Song, L.; Moon, J.J.; Sun, Z.-Y.J.; Bershteyn, A.; Hanson, M.C.; Cain, D.; Goka, S.; Kelsoe, G.; Wagner, G.; et al. Immunogenicity of Membrane-bound HIV-1 gp41 Membrane-proximal External Region (MPER) Segments Is Dominated by Residue Accessibility and Modulated by Stereochemistry. J. Boil. Chem. 2013, 288, 31888-31901. [CrossRef] [PubMed]

49. Andersen, P.H.; Nielsen, M.; Lund, O. Prediction of residues in discontinuous B-cell epitopes using protein 3D structures. Protein Sci. 2006, 15, 2558-2567. [CrossRef] [PubMed]

50. Palatnik-de-Sousa, C.B.; Soares, I.d.S.; Rosa, D.S. Editorial: Epitope Discovery and Synthetic Vaccine Design. Front. Immunol. 2018, 9, 826. [CrossRef]

51. Yasser, E.-M.; Honavar, V. Recent advances in B-cell epitope prediction methods. Immun. Res. 2010, 6, 1-9.

52. Liu, W.; Chen, Y.-H. High epitope density in a single protein molecule significantly enhances antigenicity as well as immunogenicity: A novel strategy for modern vaccine development and a preliminary investigation about B?cell discrimination of monomeric proteins. Eur. J. Immunol. 2005, 35, 505-514. [CrossRef]

53. Kapadia, C.H.; Tian, S.; Perry, J.L.; Luft, J.C.; DeSimone, J.M. Role of Linker Length and Antigen Density in Nanoparticle Peptide Vaccine. ACS Omega 2019, 4, 5547-5555. [CrossRef]

54. Liu, W.; Peng, Z.; Liu, Z.; Lu, Y.; Ding, J.; Chen, Y.-H. High epitope density in a single recombinant protein molecule of the extracellular domain of influenza A virus M2 protein significantly enhances protective immunity. Vaccine 2004, 23, 366-371. [CrossRef]

55. Bazmara, H.; Rasooli, I.; Jahangiri, A.; Sefid, F.; Astaneh, S.D.A.; Payandeh, Z. Antigenic Properties of Iron Regulated Proteins in Acinetobacter baumannii: An in Silico Approach. Int. J. Pept. Res. Ther. 2019, 25, 205-213. [CrossRef]

56. Adhikari, U.K.; Rahman, M.M. Comparative analysis of amino acid composition in the active site of nirk gene encoding copper-containing nitrite reductase (CuNiR) in bacterial spp. Comput. Boil. Chem. 2017, 67, 102-113. [CrossRef] 
57. Ke, Y.-Y.; Singh, V.K.; Coumar, M.S.; Hsu, Y.C.; Wang, W.-C.; Song, J.-S.; Chen, C.-H.; Lin, W.-H.; Wu, S.-H.; Hsu, J.T.A.; et al. Homology modeling of DFG-in FMS-like tyrosine kinase 3 (FLT3) and structure-based virtual screening for inhibitor identification. Sci. Rep. 2015, 5, srep11702. [CrossRef] [PubMed]

58. Fernando, B.-G.; Yersin, C.-T.; José, C.-B.; Paola, Z.-S. Predicted 3D Model of the Rabies Virus Glycoprotein Trimer. BioMed Res. Int. 2016, 2016, 1-11. [CrossRef]

59. Zobayer, N.; Hossain, A.A. In silico Characterization and Homology Modeling of Histamine Receptors. J. Boil. Sci. 2018, 18, 178-191. [CrossRef]

60. Khor, B.Y.; Tye, G.; Lim, T.S.; Noordin, R.; Choong, Y.S. The Structure and Dynamics of BmR1 Protein from Brugia malayi: In Silico Approaches. Int. J. Mol. Sci. 2014, 15, 11082-11099. [CrossRef] [PubMed]

(C) 2020 by the authors. Licensee MDPI, Basel, Switzerland. This article is an open access article distributed under the terms and conditions of the Creative Commons Attribution (CC BY) license (http://creativecommons.org/licenses/by/4.0/). 